\title{
Modeling and Experimental Analysis of Cephalosporin C Acylase and Its Mutant
}

\author{
Ren $\mathrm{Yu}^{*}$
}

Key Laboratory of Ecology and Biological Resources in Yarkand Oasis at Universities under the Education Department of Xinjiang Uygur Autonomous Region, Department of Kashi Normal College, No.29. Xueyuan Road, Kashi Prefecture, Xinjiang Uygur Autonomous Region, China

\begin{abstract}
ACA) is the crucial intermediate for the synthesis of semi-synthetic antibiotics, which is currently prepared by two-step biocatalysis using D-amino acid oxidase and glutaryl-7-amino cephalosporanic acid acylase (GL-7-ACA acylase) starting from cephalosporin C (CPC). Compared with the two-step enzymatic method, one-step method is more efficient and economical. But, the available Cephalosporin C acylase (CPC acylase) always take glutaryl-7-amino cephalosporanic acid (GL-7-ACA) as their primary substrate, and have low catalytic activities towards $\mathrm{CPC}$ to be used in industry. We investigated the catalytic mechanism of CPC acylase by the sequence alignment, homology modeling, and active site analysis to a series of CPC acylases from Pseudomonas where some effective mutations have been reported for activity enhancement. Two CPC acylases coded by the genes acyII and S12 are studied intensively for the interaction between the amino acid residues in the activity region and the substrate CPC based upon the complex structure obtained from the homology modeling and molecular docking. Furthermore, the catalytic parameters of the two CPC acylases were measured experimentally in order to corroborate the modeling analysis and propose potential designing strategy for improvement of enzymic activity.
\end{abstract}

Keywords: 7-amino-cephalosporanic acid, Cephalosporin C, Cephalosporin C acylase, Homology modeling, Molecular docking, Protein designing.

\section{INTRODUCTION}

The semi-synthetic cephalosporins became the popular antibiotics due to their excellent characteristics such as broad spectrum, low toxicity, and resistance to the $\beta$-lactamase and made tremendous contribution to fight with bacterial infection [1]. The semi-synthetic cephalosporin is synthesized from the intermediate 7-amino cephalosporanic acid (7ACA), which shares more than $40 \%$ of the global antiinfective market [2].

Currently, 7-ACA used for the semi-synthetic cephalosporin antibiotics is produced from Cephalosporin $\mathrm{C}$ (CPC) by either the chemical or the enzymatic methods. Among them, the two-step enzymatic method, is becoming dominant gradually $[3,4]$ because of its friendship to environment. However, this process is expensive and can't completely satisfy the industrial production. In comparison, the one-step enzymatic method is efficient and has been studied intensively [2]. Researchers separated CPC acylase from the micro-organisms which could convert CPC into 7-ACA directly [5-9]. But, the application of the wild strain was inconvenient [3] and the question was that CPC acylases

\footnotetext{
*Address correspondence to this author at the Key Laboratory of Ecology and Biological Resources in Yarkand Oasis at Universities under the Education Department of Xinjiang Uygur Autonomous Region, Department of Biological Science, Kashi Normal College, No.29. Xueyuan Road, Kashi Prefecture, Xinjiang Uygur Autonomous Region, China;

Tel: 0086-15739952831; E-mail: renyu1020@yahoo.com.cn
}

used glutaryl-7-amino cephalosporanic acid (GL-7-ACA) as their primary substrate normally and their specificity towards CPC was too low to be used in industry $[10,11]$. Some mutations with improved activity towards CPC have been developed By protein engineering of the CPC acylases Oh et al. [12] found that the deacylation activity of the mutation Q50 $3 \mathrm{M}-\mathrm{Y} 149 \alpha \mathrm{K}-\mathrm{F} 177 \beta \mathrm{G}$ toward CPC was improved by $790 \%$. Pollegioni et al. [2] used the approach of the the homology modeling combined with the site-directed mutagenesis to produce the A215Y-H296S-H309S mutation which had slightly higher activity towards CPC $(3.8 \mathrm{U} / \mathrm{mg}$ protein) than to GL-7-ACA (2.7 U/mg protein). Ishii et al. [13] found that the mutation M269W caused the 1.6-fold increase of the specific activity against CPC and observed that the minor change of conformation induced by the mutation increased the stability of the enzyme-substrate complex. Saito et al. [14] suggested that Met164 was located in the binding region in the interior surface of the CPC acylase for recognition of the substrate and found that the mutantion M164L enhanced CPC acylase activity.

In this paper, the homology modeling and the structural analysis have been applied into a series of CPC acylyses on which some mutations have been reported for improved activity (Table 1). The two genes named acy II and S12 coding the CPC acylase Acy II and its mutation named S12 respectively. acy II and S12 were constructed into pET28a and expressed in the E.coli BL21(DE3) for experimental analysis.

2013 Bentham Open 
Table 1. Reported CPC Acylases and Mutants from Pseudomonas sp.

\begin{tabular}{|c|c|c|}
\hline Author, Year & Modified Amino Acids & Enzyme Activity \\
\hline Ishii et al., 1994 & Y270F & Decreased activity \\
\hline Nobbs et al., 1994 & Y270F & Decreased activity \\
\hline Ishii et al., 1995 & M269Y or F & 1.6-fold and 1.7-fold increase \\
\hline Saito et al, $1996^{\mathrm{b}}$ & M164L & Enhance activity \\
\hline \multirow[t]{2}{*}{ Saito et al., $1996^{\mathrm{a}}$} & A271Y & Increase 1.2 -fold \\
\hline & $\mathrm{Y} 270 \mathrm{~A}$ or $\mathrm{Y} 270 \mathrm{~F}$ or $\mathrm{Y} 270 \mathrm{~L}$ or $\mathrm{Y} 270 \mathrm{~S}$ & decrease \\
\hline \multirow[t]{2}{*}{ Yamada et al., 1996} & C199S,C277S,C305S,C391S,C496S & decrease \\
\hline & C305S-M269S & 1.6-fold increase \\
\hline
\end{tabular}

${ }^{a}[14] ;[26]$

With the approach of the homology modeling and the experimental analysis, we established the preliminary knowledge about discovering the potentially efficient CPC acylase in silico, which played the important role for the one-step preparation of 7-ACA for CPC enzymatically.

\section{MATERIALS AND METHODS}

\subsection{Homology Modeling}

A series of CPC acylases originated from Pseudomonas sp. were chosen for modeling analysis in this work. Six protein templates from PDB [15] were selected to build the homology model of AcyII, i.e., Penicillin G acylase from Escherichia coli (PDB code 1e3a) sharing 36.3\% sequence similarity with AcyII; Penicillin acylase complexed with 3, 4-dihydroxyphenylacetic acid (PDB code 1ai4) sharing $37.5 \%$ similarity; Cephalosporin acylase in complex with glutaryl-7-aminocephalosporanic acid (PDB code 1jvz) sharing 37.9\% similarity; Penicillin amidohydrolase (PDB code

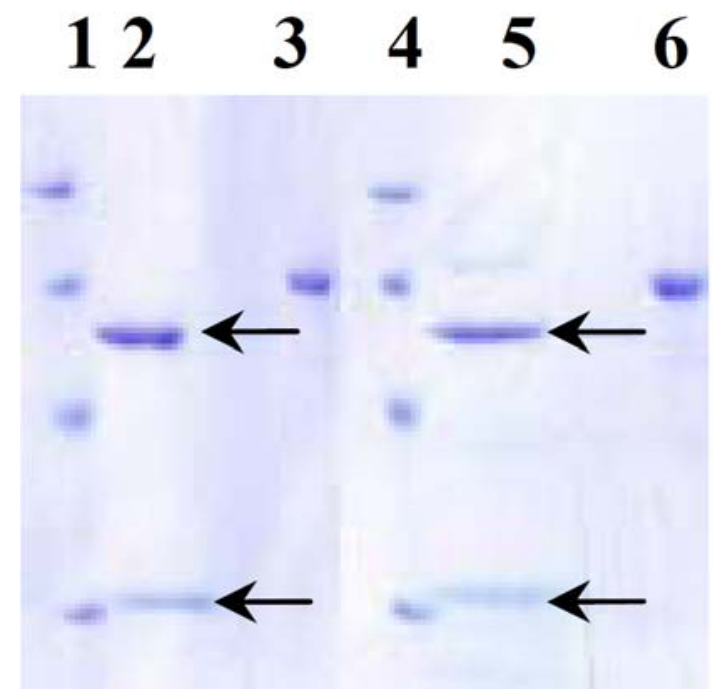

Fig. (1). SDS-PAGE of purified CPC acylase Acy II and S12 Lane 1, Molecular weight marker (from above to below: $97.2 \mathrm{kDa}$, $66.4 \mathrm{kDa}, 44.3 \mathrm{kDa}, 29.0 \mathrm{kDa})$; lane2, Acy II ; lane 3, BSA; lane 4, Marker; lane 5, S12; Lane 6, BSA.
$1 \mathrm{pnm}$ ) sharing $36.5 \%$ similarity; Glutarylamidase (PDB code 1gk1) sharing $38.5 \%$ similarity, and Penicillin G acylase from Alcaligence faecalis (PDB code $3 \mathrm{k} 3 \mathrm{w}$ ) sharing $39.4 \%$ similarity with AcyII. The result of sequence alignment was obtained by using Discovery Studio 2.1 (Accelrys, v2.0, 2009).

The model of AcyII was constructed by using the Homology Modeling Module in Accelrys Discovery Studio 2.1. The quality of the predicted model was evaluated by the Discrete Optimized Protein Energy (DOPE) by running the Verify Protein module. By the CHARMm force field [16], the conformation of amino acid residues in AcyII structural model was further modified by a standard dynamics cascade created by joining a set of steps of minimization and equilibration including minimization with steepest descent, minimization with conjugate gradient, dynamics with heating, equilibration dynamics and production dynamics. Next, the potential binding region on which the CPC was docked were identified by the Dock Ligands Module in Discovery Studio.

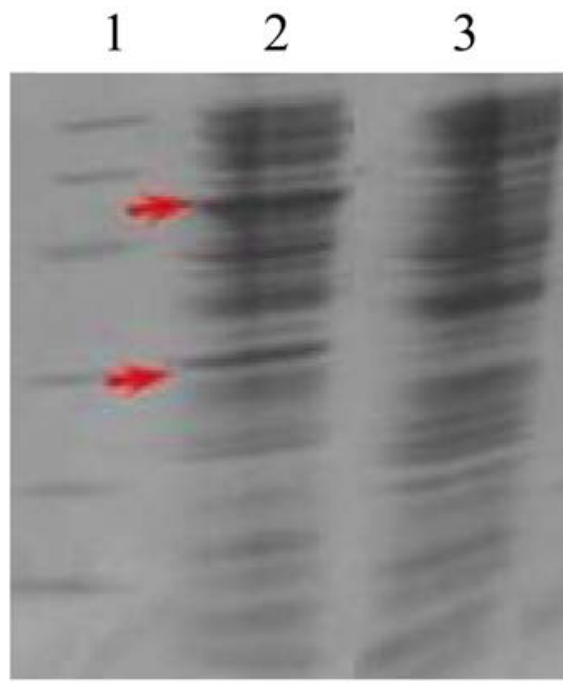

Fig. (2). Expression of CPC acylase Acy II and S12

Lane 1, Molecular weight marker (from above to below: $97.2 \mathrm{kDa}$, $66.4 \mathrm{kDa}, 44.3 \mathrm{kDa}, 29.0 \mathrm{kDa}, 20.1 \mathrm{kDa}, 14,3 \mathrm{kDa})$; lane2, Acy II ; lane 3, S12. 
After obtaining the preliminary model of AcyII-CPC complex, the PRODA, a PROtein Design Algorithmic software $[17,18]$, was applied to place the CPC on the active region under the catalytic constraints between the $\mathrm{CPC}$ and the four catalytic residues, i.e., Ser $\beta 1$, His $\beta 23$, His $\beta 70$ and Asn $\beta 242$.

\subsection{Experimental Procedures}

\subsubsection{Mutagenesis}

The gene acy II [19] was synthesized. Its mutant named S12 was obtained by the overlapping primer PCR with the substituted amino acid residues V121 $\alpha \mathrm{A}-\mathrm{G} 139 \alpha \mathrm{S}-\mathrm{F} 58 \beta \mathrm{N}-$ I75 $\beta$ T-I176 $\beta V-S 471 \beta C$. The two genes were cloned into the pET28a $(+)$ plasmids, sequenced and transformed into the E.coli BL21(DE3). For all primers, mutant positions were donoted in lowercase and the restriction sites are underlined. Backward primers designed with completely complementary role are marked with asterisk.

\section{5'-GAGCTCATGACCATGGCGGCGAA-3'*CTCGAGTACTGGTA- CCGCCGCTT}

5'-CAGGAACTGGTGCCGGCGCTCGAG-3'

*GTCCTTGACCACGGCCGCGAGCTC

5'-GCGTATgcgGCTGGAGTTAA-3'

*CGCATAcgcCGACCTCAATT

\section{5'-CGAATATagcCTGCT-3' *GCTTATAtcgGACGA \\ 5'-GCTTTCCGCATaatGCGCA-3' \\ *CGAAAGGCGTAttaCGCGT \\ 5'-CGTTTATGGATaccCAT-3' \\ *GCAAATACCTAtggGTA-3'}

5'-GGCCTGgttGATCAT-3' * *CGGACcaaCTAGTA

5'-CGCGCTGtgcCGTTAT-3'

*GCGCGACacgGCAATA

\subsubsection{Expression and Purification of AcyII and S12}

E. coli BL21(DE3) carrying the gene acy II was grown in LB medium containing $50 \mathrm{ug} / \mathrm{mL}$ kanamycin with shaking at $37^{\circ} \mathrm{C}$ overnight. A quantity of $100 \mathrm{ml}$ of fresh LB medium was inoculated with $1 \mathrm{ml}$ overnight culture and incubated with shaking to an O.D. $600 \mathrm{~nm}$ of 0.6. Then expression was induced by addition of $1 \mathrm{mmol} \mathrm{L}^{-1}$. The cell pellet of $100 \mathrm{ml}$ induced BL21(DE3) containing acy II was suspended in $100 \mathrm{mM}$ Tris- $\mathrm{HCl}$ buffer (pH8.0) and was sonicated for $20 \times 10$ s with 10 s pause at $200-300 \mathrm{w}$. The supernatant was loaded to $2 \mathrm{ml}$ Ni-NTA and eluted with $0 \mathrm{mM}$ inidazole, $50 \mathrm{mM}$ imidazole, $100 \mathrm{mM}$ imidazole, $200 \mathrm{mM}$ imidazole and $500 \mathrm{mM}$ imidazole in a succession. The target fractions were pooled and analyzed by SDS-PAGE.

\subsubsection{Assay of AcyII and S12 Activity}

Enzymic activity was determined for conversion of CPC to 7-ACA. $500 \mathrm{u} 1 \mathrm{~S} 12$ (approximately $1 \mathrm{uM}$ for CPC) was added to $500 \mathrm{ul} \mathrm{CPC}(20 \mathrm{mg} / \mathrm{ml}$ in $0.1 \mathrm{M}$ Tris/ $\mathrm{HCl}, \mathrm{pH} 8.0)$, and the mixture was incubated at $37^{\circ} \mathrm{C}$ for $8 \mathrm{~min}$. The reaction was stopped by addition of $5 \%$ acetic acid. After centrifugation $(10,000 \mathrm{rpm}, 5 \mathrm{~min})$, the formed $7-\mathrm{ACA}$ in the supernatant was determined by HPLC. One unit was defined as the amount of the enzyme liberating 1 umol 7-ACA $/ \mathrm{min}$.

\section{RESULTS AND DISCUSSION}

\subsection{Analysis of AcyII Model}

The Ramachandran diagram for the model of AcyII shows that there were $92.1 \%$ of residues falling in the allowed region, and $6.3 \%$ of residues in the marginal region. The remaining $1.6 \%$ of residues in the disallowed region was mostly far from the active region. In addition, the Verify Score of the model predicted by Accelrys Discovery Studio 2.1 was 178.74 while the Verify Expected High and Low Scores were 214.58 and 96.56, respectively. (The Verify Expected High Score is the score that would be expected for a correct structure having this sequence length, based on a statistical analysis of high-resolution structures in the Protein Data Bank. The Verify Expected Low Score is $45 \%$ of the first and is a score that is typical of grossly misfolded structures having this sequence length. The higher the Verify Score is and the more correct the structure is). These two types of data confirmed the reliability and correctness of the model of AcyII.

The homologous model and the active region of AcyII shown in Fig. (3a,b) and Fig. (4) were consistent with those of other acylases described by Fritz-Wolf et al. [20]. These acylases used conserved Ser1 $\beta$ as catalytic residue which was characteristic of the N-terminal hydrolase family. For Ser $1 \beta$, its hydroxyl group was fixed by the conserved His $23 \beta$ and its $\mathrm{NH}$ group formed a hydrogen bond with His $23 \beta$. The $\mathrm{NH}$ groups from the backbone of His $70 \beta$ and side chain of Asn $242 \beta$ formed the oxyanion hole for carboxyl group of CPC. The sequence alignment (Fig. 5a) for different acylases, i.e., AcyII, CAD [21], and PGA [22] implied that those catalytic residues were conserved, just His $70 \beta$ was variable. The role of His $70 \beta$ was to stabilize the hydrogen bond by using its backbone, which was consistent with the corresponding residues in PGA and CAD whose crystal structures were known. In the binding region, oxygen atoms from the carboxylate group of CPC interacted with Arg24 $\beta$, Tyr32 $\beta$ and His57 $\beta$. The amino adipyl moiety of CPC was stabilized

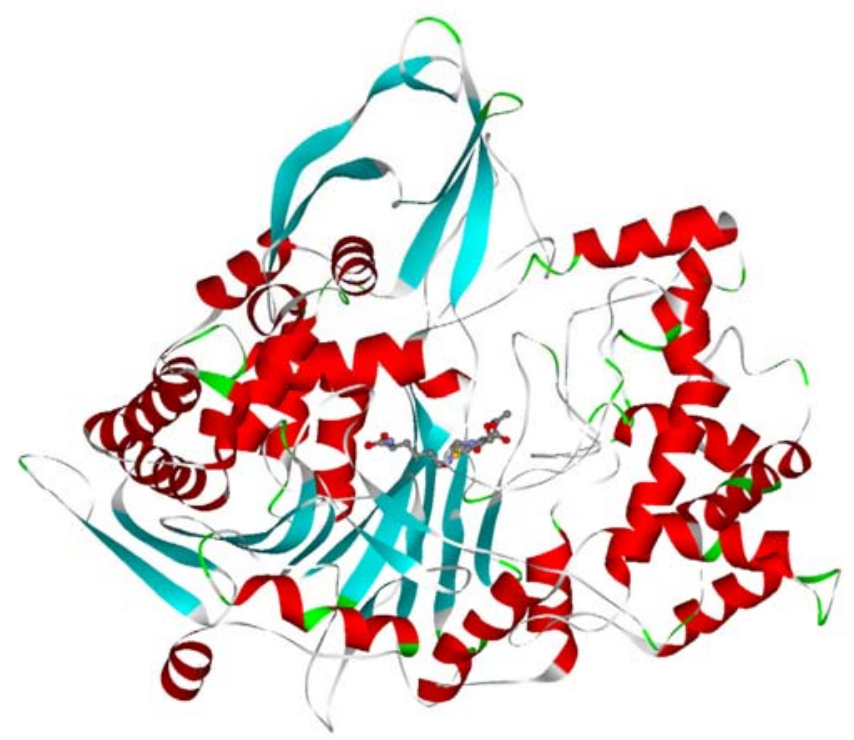

Fig. (3a). The model of AcyII, in which the substrate CPC is shown in ball and stick mode. 


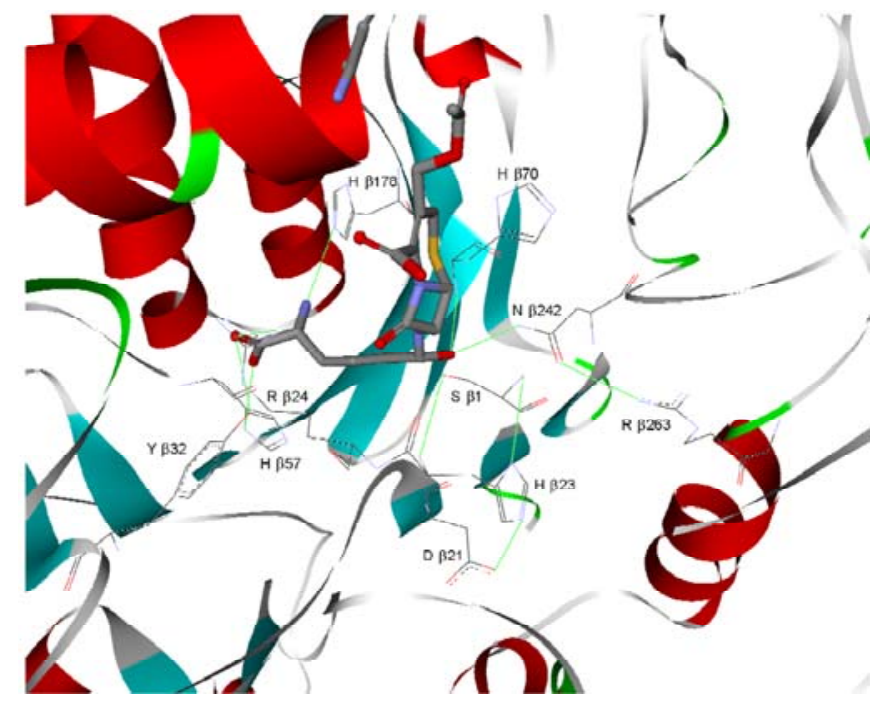

Fig. (3b). View of the active region with substrate for AcyII based on model.

The key catalytic and binding residues are shown in thin line model while the substrate CPC is shown in stick model. The hydrogen bonds are shown in green lines.

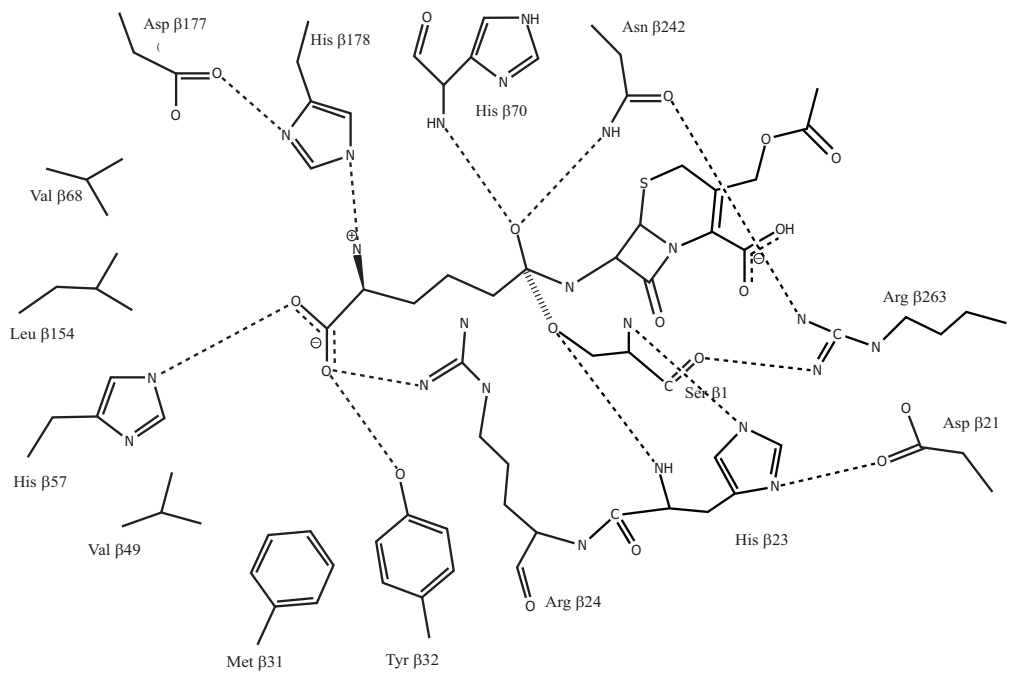

Fig. (4). Schematic drawing of the active amino acid residues for AcyII based on model.

by the formed hydrogen bond with His $178 \beta$ which simultaneously interacted with Asp177 $\beta$ stated by Fig. (4).

N176 was found active to CPC. Since AcyII and N176 shared a sequence similarity up to $93.5 \%$, shown by Fig. (5b) for sequence alignment, it was reasonable to consider that N176 and S12 shared the high structural similarity. So the analysis of mutation of N176 could be based on the same model of AcyII.

\subsection{Structural Analysis of Active Site Mutations of N176}

Ishii et al. [23] reported that the mutation of $\mathrm{W} 32 \beta \mathrm{F}$ led to the decreasing of $k_{c a t}$ to about $50 \%$. Nobbs et al. [24] reported the mutation of $\mathrm{W} 32 \beta \mathrm{L}$ whose $k_{\text {cat }}$ was reduced by $32.2 \%$. Another effort was made by Saito et al. [25] who changed Tyr32 $\beta$ to Ala/ Ser, which resulted in the decreasing of specific activity. These three studies confirmed the key binding effect of Tyr32 $\beta$ which interacted with the carboxyl group of CPC shown in Fig. (6a). Either of the mutations to
Phe $32 \beta$ or Leu32 $\beta$ demolished the original hydrogen bond with the carboxyl group on CPC, thus led to the decreasing of binding effect with CPC. The decreased activity from mutation W32 $\beta \mathrm{S}$ was possibly that the distance between the polar groups on Ser32 $\beta$ and CPC was too short to form hydrogen bond.

The mutations from Met31 to Phe31 $\beta$ or Tyr31 $\beta$ reported by Ishii et al. [13] enhanced catalytic activity by 1.6fold and 2.5-fold. The effect of these mutations is rational as the phenyl group from either phenylalanine or tyrosine could form $\pi-\pi$ conjugation with that from the neighboring Tyr32 $\beta$ and this would help the neighboring residue Tyr32 $\beta$ to maintain the suitable conformation in order to form the hydrogen bond with CPC, as that shown by Fig. (6a).

\subsection{Structural Analysis About the Mutations for AcyII}

In this paper, the mutation V121 $\alpha \mathrm{A}-\mathrm{G} 139 \alpha \mathrm{S}-\mathrm{F} 58 \beta \mathrm{N}-$ I75 $\beta$ T-I176 $\beta \mathrm{V}-\mathrm{S} 471 \beta \mathrm{C}$ named S12 was investigated. F58 $\beta \mathrm{N}$, 


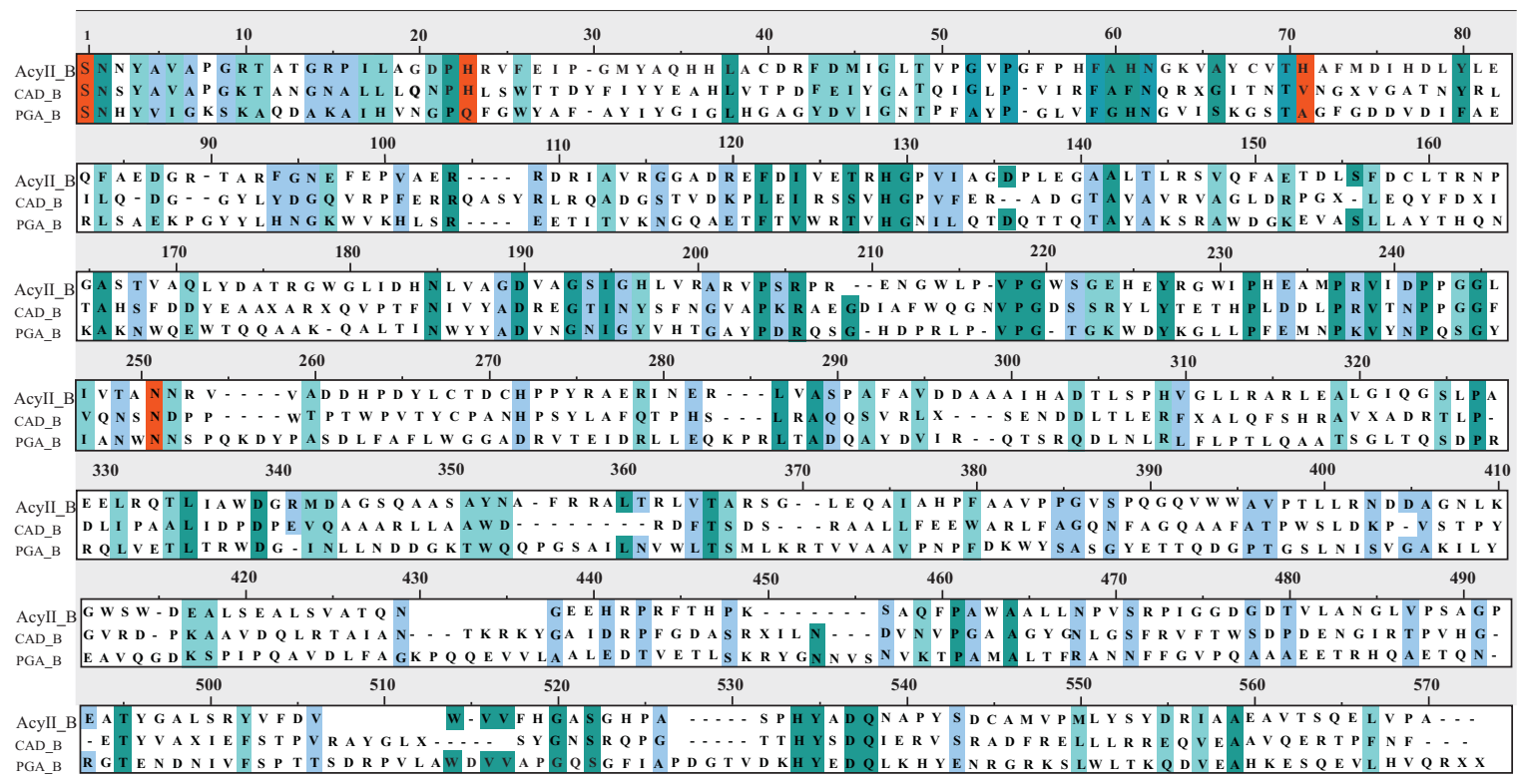

Fig. (5a). Sequences alignment of $\beta$-chains from AcyII, CAD and PGA

The four catalytic sites, i.e., Ser1 $\beta$, His $23 \beta$, His $70 \beta$ and Asn242 $\beta$ in AcyII, and their corresponding sites in CAD and PGA are shown in red.

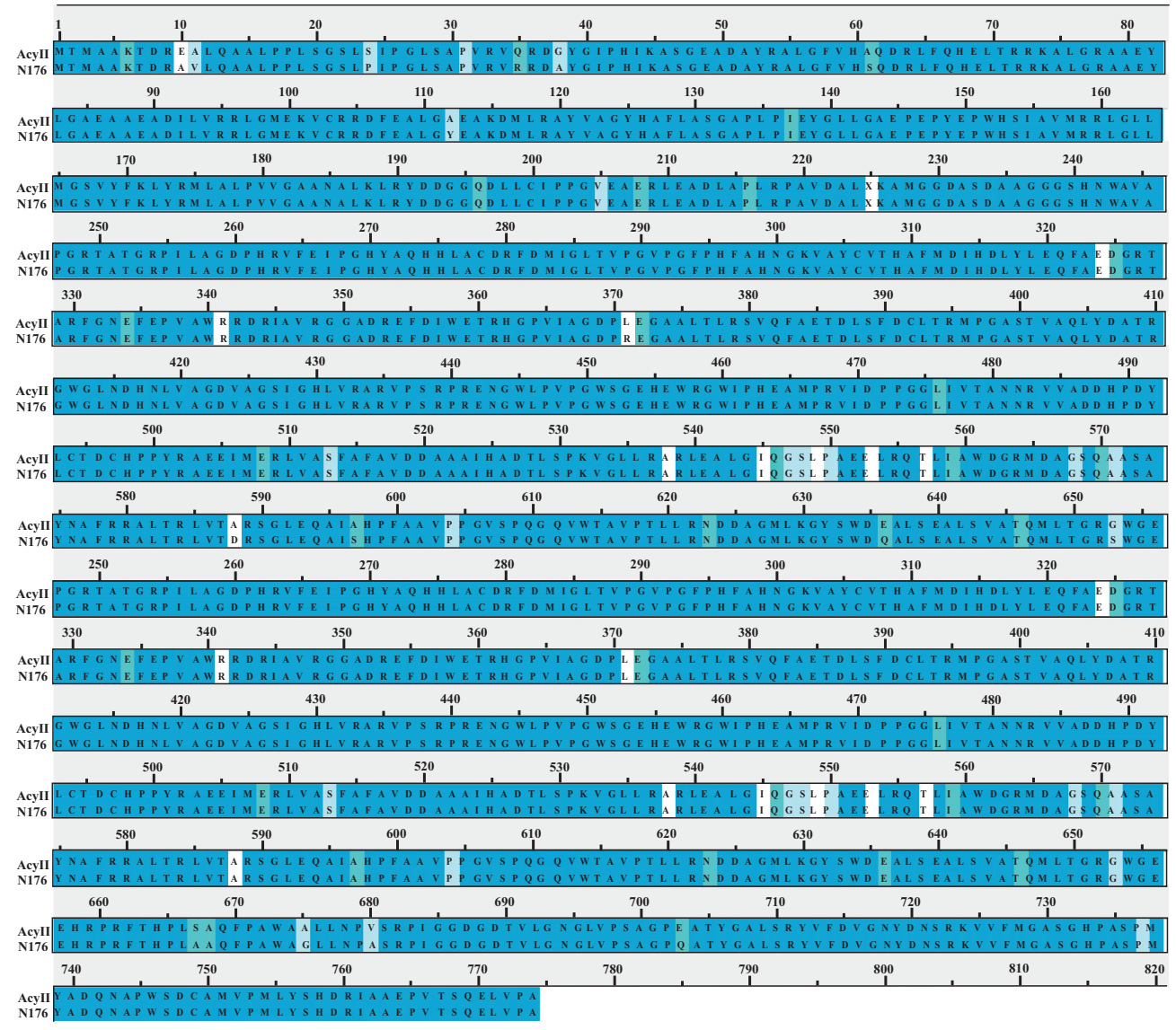

Fig. (5b). Sequence alignment of N176 and Acy II, where different residues are indicated.

I75 $\beta \mathrm{T}$ and I176 $\beta \mathrm{V}$ were close to the active region according to the model. For the mutation F58 $\beta$ N firstly, Asn $58 \beta$ had the polar carboxyl group which was different from the original hydrophobic Phe $58 \beta$, thus could form hydrogen bond with the glyoxaline group on His $57 \beta$ and stabilize the car- boxyl group of $\mathrm{CPC}$ by the $\mathrm{N}-\mathrm{O}$ hydrogen bond, as that shown in Fig. (6a). Secondly, the phenyl group from Phe $58 \beta$ would clash with CPC because of its larger side chain and lead to increasing energy and decreasing stability compared with the mutation Asn $58 \beta$. 

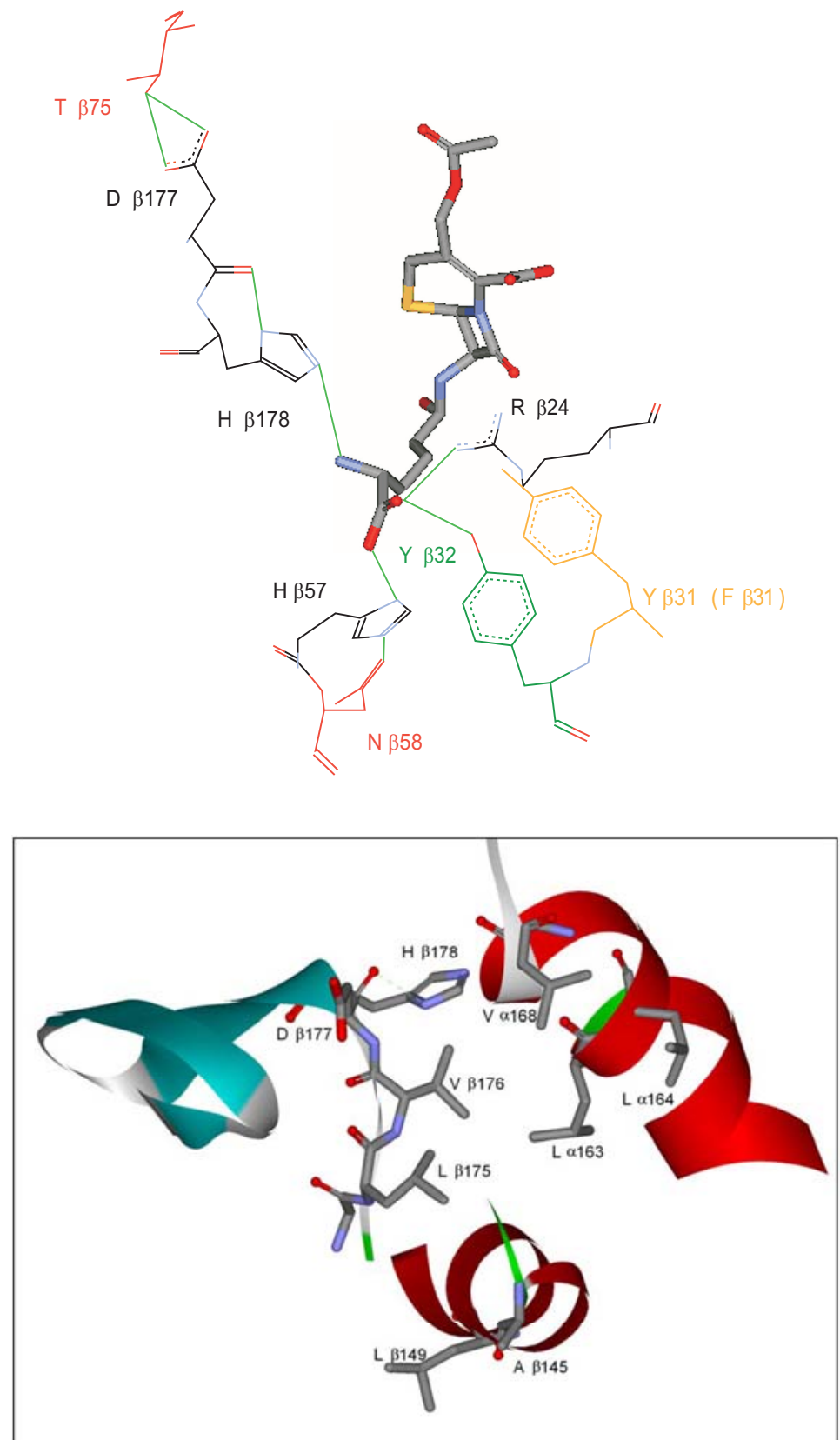

Fig. (6). View of the mutations at active sites of AcyII

(a). The two residues, i.e., Asn58 $\beta$ and Thr75 $\beta$, are colored by red. The residues Tyr $31 \beta$ or Phe $31 \beta$ is colored by orange. The important binding residue Tyr $32 \beta$ which interacts with the CPC is colored by dark green. The other residues are shown in line mode while CPC is in stick mode; (b). The residue Val176 $\beta$ with its neighboring non-polar residues

As to the mutation $\mathrm{I75} \beta \mathrm{T}$, the non-polar side residue Ile $75 \beta$ was replaced by the polar residue $\mathrm{Thr} 75 \beta$ which was located in a loop area in the binding region shown by Fig. (6a). The carboxyl group on Thr75 $\beta$ could stabilize the neighboring Asp177 $\beta$ by forming two hydrogen bonds. The C-O group on Asp177 $\beta$ interacted with ND1 on His178 $\beta$ which fixed the amino adipyl moiety in CPC. These structural interactions implied that the mutation from Ile $75 \beta$ to Thr75 $\beta$ was more favorable because the polar side chain contributed to the stability of the binding region by supplying additional hydrogen bonds.

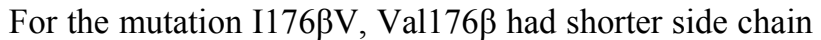
than that of Ile176 $\beta$, which could avoid the side chain clashes with the neighboring residues, such as Leu163 $\alpha$ and Leu175 $\beta$, as that shown in Fig. (6b). And Val176 $\beta$ was located in a loop region near the two important binding residues, i.e., Asp175 $\beta$ and His176 $\beta$, which interacted with the amino adipyl moiety in $\mathrm{CPC}$, so the effect of reducing spatial clashes was beneficial to stabilize the important interactions for binding.

In addition, the three residues, V121 $\alpha \mathrm{A}, \mathrm{G} 139 \alpha \mathrm{S}$ and $\mathrm{S} 471 \beta \mathrm{C}$ were far from the active region. The mutations $\mathrm{V} 121 \alpha \mathrm{A}$ and $\mathrm{G} 139 \alpha \mathrm{S}$ were related to expression level as 
Table 2. Catalytic Kinetic Parameters for CPC Acylase Acy II and S12

\begin{tabular}{|c|c|c|c|}
\hline Protein & $\boldsymbol{K}_{\boldsymbol{m}}(\mathbf{m M})$ & $\boldsymbol{k}_{\text {cat }}\left(\mathbf{s e c}^{-1}\right)$ & $\boldsymbol{k}_{\text {cat }} / \boldsymbol{K}_{m}\left(\mathbf{s e c}^{-1}\left(\mu \mathbf{M}^{-1}\right)\right.$ \\
\hline \hline Acy II & 23.71 & 7.622 & 0.321 \\
\hline S12 & 15.26 & 14.03 & 0.919 \\
\hline
\end{tabular}

Specific activities for Acy II and S12 are measured to be 2.868 unit/mg proteins and 6.011 units/mg proteins for CPC, respectively (pH8.0). Kinetic parameters were calculated from Lineweaver-Burk plots of the primary velocity of 7-ACA transformed directly from CPC $(2.1,4.2,6.3,8.4,10.5$ and $20.9 \mathrm{mM})$ in the presence of Acy II and $\mathrm{S} 12(1 \mu \mathrm{M})$ at $37^{\circ} \mathrm{C}$ for $8 \mathrm{~min}$.

shown in Fig. (2) [26]. The mutation S471ßC gave S12 the product inhibition as Pollegioni's report [2].

\subsection{Experimental Analysis About Mutation of AcyII}

From the SDS-PAGE results shown in Fig. (1), we observed that both of the CPC acylase AcyII and S12 were expressed with the MW approximately $87 \mathrm{kDa}$ and was composed of two subunits, the $58 \mathrm{kDa} \alpha$-subunit and the $25 \mathrm{kDa} \beta$-subunit, which was consistent with that reported by Mstsuda et al. [27]. The expression levels of AcyII and S12 were measured to be $322 \mathrm{U} / \mathrm{L}$ and $291 \mathrm{U} / \mathrm{L}$, respectively, shown in Fig. (2). The specific activities of AcyII and S12 were measured and the results were shown in Table 2. And we could see that the specific activity of $\mathrm{S} 12$ was 2-fold higher than that of AcyII and it reaches $6.011 \mathrm{U} / \mathrm{mg}$ protein. The catalytic parameters $K_{m}, k_{c a t}$ and $k_{c a t} / K_{m}$ of AcyII and S12 were determined by Lineweaver-Burk plot method. The $k_{\text {cat }} / K_{m}$ of S12 was higher than that of AcyII, which indicated that the mutation of six amino acid residues increased the catalytic efficiency. The result was similar to that of N176 [14]. Combined with the theoretical analysis based on structural modeling presented in Section 3.3, it was implied that the mutations around the active region, i.e., F58 $\beta$ N- I75 $\beta$ TI176 $\beta \mathrm{V}$, enhanced the binding capability between the enzyme and the transition state of the substrate instead of substrate itself since S12 had larger $K_{m}$ but higher $k_{\text {cat }}$. By virtue of the transition state theory for enzyme catalysis [28], the enhancement of binding between enzyme and the transition state of substrate reduced the activation energy and led to the increase of turnover number, i.e., $k_{c a t}$. Because the structures between substrate and its transition state was different, the stronge binding capability between enzyme and transition state of the substrate increased the dissociation reaction between enzyme and the substrate, which could be certificated by the Michaelis constant, i.e., $K_{m}$, shown in Table 2.

\section{CONCLUSION}

In this work, Acy II from Pseudomonas sp. and the mutation named S12 were structurally modeled and experimentally characterized in order to investigate their catalytic mechanism for further designing highly efficient enzyme to the one-step preparation of 7-ACA from CPC. With the methods of sequence alignment, homology modeling, and molecular docking, the structures of the active region of CPC acylase and the complex were obtained and the mutations around active site were analyzed based on intermolecular binding interaction, including steric hindrance, hydrogen bonding stabilization, solvation, and electrostatic contribution. The further experimentally measured catalytic parame- ters, i.e., $k_{c a t}$ and $K_{m}$, for CPC acylase AcyII and S12 confirmed the predicted model and provided strong evidence that the mutations around active region for increased activity would contribute to binding reaction between enzyme and the transition state substrate and decreasing activation energy for reaction. This implied that further designing of highly efficient CPC acylase in silico should focus on the amino acid sites for stronger transition state binding capability.

\section{CONFLICT OF INTEREST}

The authors confirm that this article content has no conflicts of interest.

\section{ACKNOWLEDGEMENT}

Y.R sincerely appreciates the financial support from the National Science Foundation of China and the National High Technology Research and Development (863) Program of China. Y.R thanks to Dr. Yushan Zhu and Dr. Jianan Zhang for their support and helpful discussion throughout the experimental course of this work.

\section{REFERENCES}

[1] Stefanie S, Christian MD, Jeffrey S, et al. The cephalosporin antibiotics. Prim Care Update for Ob Gyns 1997; 4: 168-74.

[2] Pollegioni L, Lorenzi S, Rosini E, et al. Evolution of an acylase active on cephalosporin C. Protein Sci 2005; 14: 3064-76.

[3] Shibuya Y, Matsumoto K, Fuji T, et al. Isolation and properties of 7ß-(4-carboxy butanamido) cephalosporanic acid acylase producing bacteria. Agric Biol Chem 1981; 45: 1561-7.

[4] Ichikawa S, Murai Y, Yamamoto S, et al. The isolation and properties of Pseudomonas mutants with all enhanced productivity of 73-(4carboxybutanamido) cephalosporinic acid acylase. Agric Biol Chem 1981; 45: 2225-9.

[5] Zhu SC, Yang YL, Zhao GP, et al. A rapid and specific method to screen environmental microorganisms for cephalosporin acylase activity. J Microbiol Methods 2003; 54: 131-5.

[6] Kim DW, Yoon KH. Cloning and high expression of glutaryl 7aminocephalosp- oranic acid acylase gene from Pseudomonas diminuta. Biotechnol Lett 2001; 23: 1067-71.

[7] Aramori I, Fukagawa M, Tsumura M. Cloning and nucleotide sequencing of a novel 7 $\beta$-(4-carboxybutanamido) cephalosporanic acid acylase gene of Bacillus laterosporus and its expression in Escherichia coli and Bacillus subtilis. J Bacteriol 1991훌 173: 7848-55.

[8] Aramori I, Fukagawa M, Tsumura M, et al. Isolation of soil strains producing new cephalosporin acylases. J Ferment Bioeng 1991 ; 72 : 227-31.

[9] Aramori I, Fukagawa M, Tsumura M, et al. Cloning and nucleotide sequencing of new glutaryl 7-ACA and cephalosporin $\mathrm{C}$ acylase genes from Pseudomonas strains. J Ferment Bioeng 1991'; 72: 23243.

[10] Zhang QJ, Xu WX. Morphological, physiological and enzymatic characteristics of cephalosporin acylase-producing Arthrobacter strain 45-8A. Arch Microbiol 1993; 159: 392-5. 
[11] Deshpands BS, Ambedkar SS, Shewale JG. Cephalosporin C acylase and penicillin V acylase formation by Aeromonas sp.ACY95. World J Microbiol Biotechnol 1998; 12: 373-8.

[12] Oh B, Kim M, Yoon J, et al. Deacylation activity of cephalosporin acylase to cephalosporin $\mathrm{C}$ is improved by changing the side-chain conformations of active-site residues. Biochem Biophys Res Commun 2003; 310: 19-27.

[13] Ishii Y, Saito Y, Fujimura T, et al. High-level production, chemical modification and site-directed mutagenesis of a cephalosporin $\mathrm{C}$ acylase from Pseudomonas strain N176. Eur J Biochem 1995; 230: $773-8$.

[14] Saito Y, Ishii $Y$, Fujimura $T$, et al. Protein engineering of a cephalosporin C acylase from Pseudomonas strain N176. Ann NY Acad Sci $1996^{\text {b }} ; 782: 226-40$.

[15] Berman HM, et al. The Protein Data Bank. Acta Crystallogr D Biol. Crystallogr 2002; 58: 899-907.

[16] Brooks BR, Bruccoleri RE, Olafson BD, et al. CHARMM: A Program for Macromolecular Energy, Minimization, and Dynamics Calculations. J Comput Chem 1983; 4: 187-217.

[17] Zhu Y. Mixed-integer linear programming algorithm for a computational protein design problem. Ind Eng Chem Res 2007; 46: 839-45.

[18] Luo W, Pei J, Zhu Y. A fast protein-ligand docking algorithm based on hydrogen bond matching and surface shape complementarity. J Mol Model 2010; 16: 903-13.

[19] Matsuda A, Toma K, Komatsu K. Nucleotide sequences of the genes for two distinct cephalosporin acylases from a Pseudomonas strain. J Bacteriol 1987 ; 169 : 5821-6.
[20] Fritz-Wolf K, Koller K-P, Lange G, et al. Structure-based prediction of modifications in glutarylamidase to allow single-step enzymatic production of 7-aminocephalosporanic acid from cephalosporin C. Protein Sci 2002; 11: 92-103.

[21] Kim Y, Yoon K.-H, Khang Y, et al. The 2.0A crystal structure of cephalosporin acylase. Structure 2000; 8: 1059-68.

[22] Duggleby HJ, Tolley SP, Dodson EJ, et al. Penicillin acylase has a single amino-acid catalytic center. Nature 1995; 373: 264-8.

[23] Ishii Y, Saito Y, Sasaki H, et al. Affinity labelling of cephalosporin C acylase from Pseudomonas sp. N176 with a substrate analogue, $7 \beta-$ (6-bromohexanoylamido) cephalosporanic acid. J Ferment Bioeng 1994; 77: 598-603.

[24] Nobbs TJ, Ishii Y, Fujimura T, et al. Chemical modification and sitedirected mutagenesis of tyrosine residues in cephalosporin $\mathrm{C}$ acylase from Pseudomonas strain N176. J Ferment Bioeng 1994; 77:604-9.

[25] Saito Y, Fujimura T, Ishii Y, et al. Oxidative Modification of a Cephalosporin C Acylase from Pseudomonas Strain N176 and SiteDirected Mutagenesis of the Gene. Appl Environ Microbiol 1996; 62: 2919-25.

[26] Saito Y, Ishii Y, Fujimura T, et al. Protein engineering of a cephalosporin C acylase from Pseudomonas strain N176. Ann N Y Acad Sci 1996; 15:226-40.

[27] Matsuda A, Matsuyama K, Yamamoto K, et al. Cloning and characterization of the genes for two distinct cephalosporin acylases from a Pseudomonas strain. J Bacteriol 1987a; 169: 5815-20.

[28] Fersh F. Structure and mechanism in protein science. New York: W. H. Freeman and Company 1999.

Received: August 18, 2013

Revised: August 22, 2013

Accepted: August 22, 2013

(C) Ren Yu; Licensee Bentham Open.

This is an open access article licensed under the terms of the Creative Commons Attribution Non-Commercial License (http://creativecommons.org/licenses/ by-nc/3.0/) which permits unrestricted, non-commercial use, distribution and reproduction in any medium, provided the work is properly cited. 